\title{
Long-term GnRH agonist therapy before in vitro fertilisation (IVF) for improving fertility outcomes in women with endometriosis
} (Review)

Georgiou EX, Melo P, Baker PE, Sallam HN, Arici A, Garcia-Velasco JA, Abou-Setta AM, Becker C, Granne IE

Georgiou EX, Melo P, Baker PE, Sallam HN, Arici A, Garcia-Velasco JA, Abou-Setta AM, Becker C, Granne IE.

Long-term GnRH agonist therapy before in vitro fertilisation (IVF) for improving fertility outcomes in women with endometriosis.

Cochrane Database of Systematic Reviews 2019, Issue 11. Art. No.: CD013240.

DOI: 10.1002/14651858.CD013240.pub2.

www.cochranelibrary.com 
[Intervention Review]

\section{Long-term GnRH agonist therapy before in vitro fertilisation (IVF) for improving fertility outcomes in women with endometriosis}

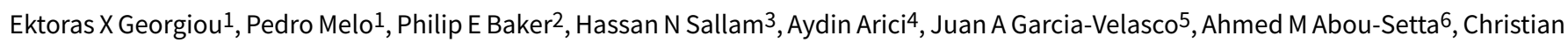
Becker? ${ }^{7}$ Ingrid E Granne ${ }^{7}$

1Buckinghamshire Hospitals NHS Trust, Aylesbury, UK. ${ }^{2}$ Academic Centre, Oxford University Hospitals NHS Trust, Oxford, UK. ${ }^{3}$ Obstetrics and Gynaecology, Alexandria University, Alexandria, Egypt. ${ }^{4}$ Reproductive Endocrinology Section, Yale University, New Haven, Connecticut, USA. 5 Instituto Valenciano de Infertilidad Madrid, IVI Madrid, Madrid, Spain. ${ }^{6}$ Knowledge Synthesis Platform, George and Fay Yee Centre for Healthcare Innovation, University of Manitoba, Winnipeg, Canada. ${ }^{7}$ Nuffield Department of Women's \& Reproductive Health, University of Oxford, Oxford, UK

Contact address: Ektoras X Georgiou, Buckinghamshire Hospitals NHS Trust, Aylesbury, UK. hgeorgiou@gmail.com.

Editorial group: Cochrane Gynaecology and Fertility Group

Publication status and date: New, published in Issue 11, 2019.

Citation: Georgiou EX, Melo P, Baker PE, Sallam HN, Arici A, Garcia-Velasco JA, Abou-Setta AM, Becker C, Granne IE. Long-term GnRH agonist therapy before in vitro fertilisation (IVF) for improving fertility outcomes in women with endometriosis. Cochrane Database of Systematic Reviews 2019, Issue 11. Art. No.: CD013240. DOI: 10.1002/14651858.CD013240.pub2.

Copyright @ 2019 The Cochrane Collaboration. Published by John Wiley \& Sons, Ltd.

\section{A B S T R A C T}

\section{Background}

Endometriosis is known to have an impact on fertility and it is common for women affected by endometriosis to require fertility treatments, including in vitro fertilisation (IVF) or intracytoplasmic sperm injection (ICSI), to improve the chance of pregnancy. It has been postulated that long-term gonadotrophin-releasing hormone $(\mathrm{GnRH})$ agonist therapy prior to IVF or ICSI can improve pregnancy outcomes. This systematic review supersedes the previous Cochrane Review on this topic (Sallam 2006).

\section{Objectives}

To determine the effectiveness and safety of long-term gonadotrophin-releasing hormone $(\mathrm{GnRH})$ agonist therapy (minimum 3 months) versus no pretreatment or other pretreatment modalities, such as long-term continuous combined oral contraception (COC) or surgical therapy of endometrioma, before standard in vitro fertilisation (IVF) or intracytoplasmic sperm injection (ICSI) in women with endometriosis.

\section{Search methods}

We searched the following electronic databases from their inception to 8 January 2019: Cochrane Gynaecology and Fertility Specialised Register of Controlled Trials, CENTRAL via the Cochrane CENTRAL Register of Studies ONLINE (CRSO), MEDLINE, Embase, PsycINFO, Cumulative Index to Nursing and Allied Health Literature (CINAHL). We searched trial registries to identify unpublished and ongoing trials. We also searched DARE (Database of Abstracts of Reviews of Effects), Web of Knowledge, OpenGrey, Latin American and Caribbean Health Science Information Database (LILACS), PubMed, Google and reference lists from relevant papers for any other relevant trials.

\section{Selection criteria}

Randomised controlled trials (RCTs) involving women with surgically diagnosed endometriosis that compared use of any type of GnRH agonist for at least three months before an IVF/ICSI protocol to no pretreatment or other pretreatment modalities, specifically use of longterm continuous $\mathrm{COC}$ (minimum of 6 weeks) or surgical excision of endometrioma within six months prior to standard IVF/ICSI. The primary outcomes were live birth rate and complication rate per woman randomised. 


\section{Data collection and analysis}

Two independent review authors assessed studies against the inclusion criteria, extracted data and assessed risk of bias. A third review author was consulted, if required. We contacted the study authors, as required. We analysed dichotomous outcomes using Mantel-Haenszel risk ratios (RRs), 95\% confidence intervals ( $\mathrm{Cls}$ ) and a fixed-effect model. For small numbers of events, we used a Peto odds ratio (OR) with $95 \% \mathrm{Cl}$ instead. We analysed continuous outcomes using the mean difference (MD) between groups and presented with $95 \% \mathrm{Cls}$. We studied heterogeneity of the studies via the $1^{2}$ statistic. We assessed the quality of evidence using GRADE criteria.

\section{Main results}

We included eight parallel-design RCTs, involving a total of 640 participants. We did not assess any of the studies as being at low risk of bias across all domains, with the main limitation being lack of blinding. Using GRADE methodology, the quality of the evidence ranged from very low to low quality.

\section{Long-term GnRH agonist therapy versus no pretreatment}

We are uncertain whether long-term GnRH agonist therapy affects the live birth rate (RR $0.48,95 \% \mathrm{Cl} 0.26$ to $0.87 ; 1 \mathrm{RCT}, \mathrm{n}=147 ; \mathrm{I}$ not calculable; very low-quality evidence) or the overall complication rate (Peto OR $1.23,95 \% \mathrm{Cl} 0.37$; to $4.14 ; 3 \mathrm{RCTs}, \mathrm{n}=318$; $\mathrm{I}^{2}=73 \%$; very low-quality evidence) compared to standard IVF/ICSI. Further, we are uncertain whether this intervention affects the clinical pregnancy rate (RR $1.13,95 \% \mathrm{Cl} 0.91$ to $1.41 ; 6 \mathrm{RCTs}, \mathrm{n}=552, \mathrm{l} 2=66 \%$; very low-quality evidence), multiple pregnancy rate (Peto $\mathrm{OR} 0.14,95 \% \mathrm{Cl} 0.03$ to $0.56 ; 2$ RCTs, $n=208, I^{2}=0 \%$; very low-quality evidence), miscarriage rate (Peto OR $0.45,95 \% \mathrm{Cl} 0.10$ to $2.00 ; 2 \mathrm{RCTs}, \mathrm{n}=208 ; \mathrm{I}^{2}=0 \%$; very low-quality evidence), mean number of oocytes (MD $0.72,95 \% \mathrm{Cl} 0.06$ to $1.38 ; 4 \mathrm{RCTs}, \mathrm{n}=385 ; \mathrm{I}^{2}=81 \%$; very low-quality evidence) or mean number of embryos (MD $-0.76,95 \% \mathrm{Cl}-1.33$ to $-0.19 ; 2 \mathrm{RCTs}, \mathrm{n}=267 ; \mathrm{I}^{2}=0 \%$; very low-quality evidence).

\section{Long-term GnRH agonist therapy versus long-term continuous COC}

No studies reported on this comparison.

\section{Long-term GnRH agonist therapy versus surgical therapy of endometrioma}

No studies reported on this comparison.

\section{Authors' conclusions}

This review raises important questions regarding the merit of long-term $\mathrm{GnRH}$ agonist therapy compared to no pretreatment prior to standard IVF/ICSI in women with endometriosis. Contrary to previous findings, we are uncertain as to whether long-term GnRH agonist therapy impacts on the live birth rate or indeed the complication rate compared to standard IVF/ICSI. Further, we are uncertain whether this intervention impacts on the clinical pregnancy rate, multiple pregnancy rate, miscarriage rate, mean number of oocytes and mean number of embryos. In light of the paucity and very low quality of existing data, particularly for the primary outcomes examined, further high-quality trials are required to definitively determine the impact of long-term GnRH agonist therapy on IVF/ICSI outcomes, not only compared to no pretreatment, but also compared to other proposed alternatives to endometriosis management.

\section{PLAIN LANGUAGE SUMMARY}

\section{Long-term pituitary down-regulation before in vitro fertilisation (IVF) for women with endometriosis}

\section{Review question}

We reviewed the efficacy and safety of treating women with known endometriosis (a disease characterised by the presence of endometrial tissue outside the cavity of the womb) with gonadotrophin-releasing hormone (GnRH) agonist medication for a period of three to six months prior to in vitro fertilisation (IVF) and intracytoplasmic sperm injection (ICSI). We aimed to compare this intervention to no treatment prior to IVF/ICSI, pretreatment with long-term continuous combined oral contraception (COC) or surgical treatment to remove endometriomas (cysts forming in the ovaries as a consequence of endometriosis).

\section{Background}

Many women affected by endometriosis suffer with infertility and may, as a result, seek IVF/ICSI treatment. IVF/ICSI is known to be less successful in women with endometriosis and a variety of interventions prior to IVF/ICSI have been proposed to try and improve outcomes. These include long-term GnRH agonist therapy, long-term continuous COC therapy or surgery to remove endometriomas.

\section{Study characteristics}

We found eight randomised controlled trials comparing long-term GnRH agonist therapy with no pretreatment including a total of 640 women with endometriosis prior to IVF/ICSI. The evidence is current to January 2019.

\section{Key results}

Long-term GnRH agonist therapy before in vitro fertilisation (IVF) for improving fertility outcomes in women with endometriosis (Review)

Copyright (c) 2019 The Cochrane Collaboration. Published by John Wiley \& Sons, Ltd. 
Compared to no pretreatment, we are uncertain whether long-term GnRH agonist therapy prior to IVF/ICSI in women with endometriosis affects the live birth rate. The evidence suggests that if the chance of live birth rate is assumed to be $36 \%$ with no pretreatment, the chance following long-term GnRH agonist therapy would be between $9 \%$ and 31\%. We are also uncertain whether this intervention affects complication rate, clinical pregnancy rate, multiple pregnancy rate, miscarriage rate, mean number of oocytes and mean number of embryos. No studies compared long-term GnRH agonist therapy to long-term continuous COC therapy or surgery to remove endometriomas.

\section{Quality of the evidence}

The evidence was of very low quality. The main limitations in the evidence were lack of blinding (the process where the women participating in the trial, as well as the research staff, are not aware of the intervention used), inconsistency (differences between different studies) and imprecision (random error and small size of each study). 